\title{
Microbeam radiation therapy: Clinical perspectives
}

\author{
M.A. Grotzer ${ }^{\text {a, }}{ }^{*}$, E. Schültke ${ }^{\text {b }}$, E. Bräuer-Krisch ${ }^{\text {c}}$, J.A. Laissue ${ }^{\text {d }}$ \\ a University Children's Hospital of Zurich, Steinwiesstrasse 75, CH-8032 Zurich, Switzerland \\ ${ }^{\mathrm{b}}$ Rostock University Medical Center, Department of Radiotherapy, Südring 75, 18059 Rostock, Germany \\ ${ }^{\mathrm{c}}$ European Synchrotron Radiation Facility, 71 Avenue des Martyrs, F-38043 Grenoble Cedex 9, France \\ d University of Bern, Bern, Switzerland
}

\section{A R T I C L E I N F O}

\section{Article history:}

Received 20 December 2014

Received in revised form

12 February 2015

Accepted 16 February 2015

Available online 13 March 2015

\section{Keywords:}

Radiation therapy

Brain tumour

Child

Diffuse intrinsic pontine glioma

Glioblastoma

\begin{abstract}
A B S T R A C T
Microbeam radiation therapy (MRT), a novel form of spatially fractionated radiotherapy (RT), uses arrays of synchrotron-generated X-ray microbeams (MB). MRT has been identified as a promising treatment concept that might be applied to patients with malignant central nervous system (CNS) tumours for whom, at the current stage of development, no satisfactory therapy is available yet. Preclinical experimental studies have shown that the CNS of healthy rodents and piglets can tolerate much higher radiation doses delivered by spatially separated MBs than those delivered by a single, uninterrupted, macroscopically wide beam. High-dose, high-precision radiotherapies such as MRT with reduced probabilities of normal tissue complications offer prospects of improved therapeutic ratios, as extensively demonstrated by results of experiments published by many international groups in the last two decades. The significance of developing MRT as a new RT approach cannot be understated. Up to $50 \%$ of cancer patients receive conventional RT, and any new treatment that provides better tumour control whilst preserving healthy tissue is likely to significantly improve patient outcomes.

() 2015 Published by Elsevier Ltd on behalf of Associazione Italiana di Fisica Medica. This is an open access article under the CC BY-NC-ND license (http://creativecommons.org/licenses/by-nc-nd/4.0/).
\end{abstract}

\section{Introduction}

Despite the technical and scientific advances of radiotherapy (RT) over the past decades, only palliative therapy is available for children and adults with a number of high-grade tumours. This often only extends the survival of individual patients by a few months. As an example, diffuse intrinsic pontine gliomas, which constitute $15 \%$ of all childhood brain tumours (600 new cases/year in Europe), are inoperable. Their response to radiation and chemotherapy is only transient, with patients having a median overall survival of 10 months [1,2].

Microbeam radiation therapy (MRT), a novel radiotherapy method invented by Slatkin and coworkers in 1992 [3], is based on a spatial fractionation of synchrotron-generated X-ray beams. Spatial fractionation and the underlying laws of radiation physics were discovered by Alban Koehler at the beginning of the 20th century. It was used to reduce the extent of skin damage, a frequently occurring adverse effect of early radiotherapy. MRT was developed in a preclinical environment as a collaborative project involving physicists, engineers, biomedical scientists, and

\footnotetext{
* Corresponding author.

E-mail address: Michael.Grotzer@kispi.uzh.ch (M.A. Grotzer).
}

physicians, initially at the National Synchrotron Light Source at Brookhaven National Laboratory, Upton (USA), and later at the European Synchrotron Radiation Facility (ESRF), Grenoble (France). MRT, based on the spatial fractionation of kilovoltage-energy Xray beams, uses arrays of SRgenerated, collimated, planar, quasiparallel microbeams (MBs; size, approximately 25-50 $\mu \mathrm{m}$, spaced at $200-400 \mu \mathrm{m}$ on centre). The synchrotron X-ray beam is segmented into a lattice of narrow, quasi-parallel, microplanar beams, typically 25 - to $50-\mu \mathrm{m}$ wide, separated by centre-to-centre distances (c-t-c) of 200-400 $\mu \mathrm{m}$ and delivered in a single treatment session, in a scanning mode. The very high in-beam MB 'peak' dose zones, in excess of $100 \mathrm{~Gy}$, are separated by very low-dose 'valley' regions. These in-beam doses are orders of magnitude greater than those normally delivered in conventional RT. At a critical collimated beam width and separation in the order of tens and hundreds of microns, respectively, normal tissue can recover from hectogray exposure levels, which were previously considered to be lethal, whilst cancerous cells within the tumour are destroyed. Extremely high X-ray doses must be delivered at very high dose rates, within a very narrow time window, to prevent blurring of the MB tracks due to organ motion, so that the irradiation of an entire organ can be performed in a fraction of a second. The $6-\mathrm{GeV}$ synchrotron ring at the ESRF is currently the only source of synchrotron radiation in Europe that is capable of generating intense X-ray microbeams, 
having a broad photon energy spectrum and fluence rates (i.e. X-ray beam intensities) high enough to deliver high absorbed physical radiation doses to deep targets in a scanning mode where a dedicated biomedical facility is available. Together with the adequate energy spectrum, the very low divergence of such X-ray beams is also conditional for preservation of the plane parallel beams and a sharp penumbra throughout the target, which is currently only feasible at such high energy synchrotron wiggler sources.

A Swiss-based research group for MRT has been intimately associated with the inventors of MRT for its development and validation over 20 years. The extensively equipped biomedical facility (ID17) at the ESRF, which is financed by 20 countries, acts as a central synchrotron RT research facility that plays a leading role in Europe and worldwide.

\section{MRT in small animals}

Preclinical long-term experiments that involved different species such as insects [4], birds [5], rodents [6-12], and pigs [13] have revealed an extraordinary tolerance of normal organs and blood vessels exposed to fractionated radiation doses in excess of $100 \mathrm{~Gy}$ delivered by arrays of MB. This tolerance was particularly evident in suckling rats and weaning piglets, whose irradiated brains are still developing [13-16].

MRT in small animal models has achieved therapeutic ratios that clearly exceed those obtained by conventional radiography with a homogeneous dose distribution, in a range of malignancies, including gliomas, gliosarcomas, human squamous cell carcinomas, and glioblastomas. These characteristics of MRT have been extensively demonstrated by results of preclinical experiments [12,17]. Furthermore, MRT-associated bystander effects have been identified [18-21]. The tumour control of MRT has been improved by combining MRT with various compounds [22-24], radiationenhancing substances [25], gene-mediated immunoprophylaxis [26], and other adjuvant techniques. It can clearly be concluded that high-dose, high-precision radiotherapies with reduced probabilities of normal tissue complications offer prospects of improved survival outcome probability and decreased risk of therapy-related toxicity.

Several probable reasons why MRT provides a higher therapeutic index for tumours than broad beam irradiation have been elucidated, such as the following: (1) MBs produce steep dose gradients between tissue slices receiving the peak and valley doses; they have a 90\%-10\% dose fall-off, about 200 times steeper than that of a Gamma Knife [27]. The radiotoxic dose is therefore confined to a very narrow zone while the integrity and functionality of the adjacent normal tissue in the valleys between the peaks can be preserved. (2) Spatial fractionation results in a very large specific contact surface between peak and valley zones. This extended contact surface is instrumental for the repair of heavily irradiated tissues in peak regions. (3) In contrast to the high tolerance of the normal microvasculature [9] and arteries [7] to irradiation by $\mathrm{MB}$, the tumour vasculature of $9 \mathrm{~L}$ gliosarcomas in rats is selectively damaged by MRT [28] with ensuing tumour hypoxia and shrinkage. Conversely, normal brain tissues exposed to MB during MRT remain sufficiently perfused to maintain normoxia [17]. (5) MB irradiation of normal rat brains provokes proteomic responses that are indicative of oncogenesis and proteomic changes associated with bystander effects, indicative of apoptosis mediated by reactive oxygen species. Furthermore, potentially antioncogenic apoptotic proteomic changes indicate that the collective interaction of such MB irradiation-induced bystander effect proteins might confer a protective effect on normal tissues [20]. (6) Transcriptional gene expression analysis of intracerebral gliosarcomas in rats [12,29] and EM6.5 breast tumours in mice [30] have identified MRT induced immunity-related modulations, clearly different from transcriptional changes induced by unsegmented broad beams.

\section{MRT for large animals}

Although encouraging, previous preclinical results in small animals are not sufficient to justify MRT studies to advance directly to phase I human clinical trials. Before moving to human applications, MRT must be applied in therapeutic veterinary trials of larger animals such as pigs bearing intracerebral glial tumours [31], as well as companion dog and cat patients bearing spontaneous tumours. The use of larger animals in MRT studies is supported by the dimensional and physiological similarities of spontaneous tumours in dogs and cats compared to those in human malignancies, in contrast to implanted tumours of mice and rats [32-35]. The physical disadvantage of using rather low-energy photons to treat larger, deep-seated targets can be overcome by the use of conformal image-guided MRT that uses several ports.

These studies will further augment our understanding of how deeper-seated and larger tumour tissues respond to MRT and serve as an early warning system for unexpected late adverse effects. Considering that the time course of biological events is compressed in domestic and companion animals compared to humans and that the large animal phase I/II trial precedes human clinical trials by several years, one can re-assess and, if necessary, refine the treatment plan for human patients based on the results obtained in these larger animal studies.

\section{MRT for human patients}

MRT for human patients requires a careful, multi-disciplinary evaluation of epidemiological, medical, logistical, and ethical considerations, including quality of life in comparison to life span, and endpoint definitions. Candidate populations could be adult patients with glioblastoma multiforme (approximately 20,000 new cases/ year in Europe). Current standard treatment consists of surgery followed by chemoradiation and adjuvant temozolomide [36], but no standard of care exists for patients with recurrent tumours.

Paediatric patients with diffuse intrinsic pontine glioma (DIPG; approximately 600 new cases/year in Europe) would be an excellent candidate population. DIPG remains a most frustrating tumour in paediatric oncology. Because of the location of tumours and the difficulty in distinguishing tumour tissue from normal structures, surgical debulking is restricted by the substantial risk of morbidity and mortality. The mainstay of therapy for intrinsic pontine glioma has been RT. While there is evidence that conventional RT provides short-term benefits (i.e. a temporary improvement in neurological function and thus an increase in the quality of life), long-term results have been dismal and the overall survival time has not changed $[1,2]$.

To safely conduct human clinical trials with MRT, new hardware and software need to be developed and tested. A patientpositioning system for MRT is currently available for small animals and larger animals such as pigs, dogs, and cats, up to a weight of $40 \mathrm{~kg}$. Designing and building a patient-positioning system that will move a heavier human patient with the required exactness/ spatial precision are therefore necessary. The therapy accuracy system used in the large animal trials was based on computed tomographic images. For clinical trials in humans, therapy planning which incorporates magnetic resonance imaging findings is desirable, as it provides higher spatial resolution [37]. A marker system for reliable repositioning between image acquisitions and positioning for treatment could be either of the fiducial type or ensured by a stereotactic frame system. The preclinical veterinary trial in 
larger animals will improve understanding of the dosimetry, therapy planning, and therapeutic results for larger, deep-seated, spontaneous tumours.

\section{Potential impact}

The significance of developing MRT as a new RT approach cannot be understated. Up to 50\% of cancer patients receive conventional RT. Especially for patients with highly malignant brain tumours, however, the improvement in quality of life and survival time with the currently available treatment schedules is unsatisfactory; any new treatment that provides better tumour control whilst preserving healthy tissue is likely to significantly improve patient outcomes. MRT has been identified as a most promising treatment concept for patients with malignant CNS tumours for whom, at the current stage of development, no satisfactory therapy is available yet [14].

MRT research is currently only possible at few centres in the world, with mainly the Australian Synchrotron in Melbourne and the ESRF in France having active programs for developing clinical applications. The availability of world-class facilities and the associated expertise of the investigators enable studies that have the potential to substantially improve RT for cancer and advance our understanding of fundamental tumour biology. ESRF is a most adequate source when moving to human trials, as the current dose rate assures no blurring of microbeams even within a slightly moving target such as the human brain.

Once we have demonstrated both the technical feasibility of using MRT in human patients and its therapeutic efficacy, results may have a major impact on the development of MB trials by using other available sources such as proton therapy, which could equally benefit from the tissue-sparing effects of beam microfractionation. The combination of MRT with drugs and/or other substances that enhance radiation effects, at optimised and tuneable X-ray energies, as well as its combination with gene-mediated therapies must be tested in order to establish whether these lead to considerable improvements compared to conventional RT alone.

The development of compact X-ray sources (presently under development in Europe by the France-based ThomX project), through which MRT may possibly be implemented, could make this promising technique available to a wider medical community.

\section{Acknowledgements}

The authors are supported by the COST-Action TD1205.

\section{References}

[1] Khatua S, Moore KR, Vats TS, Kestle JR. Diffuse intrinsic pontine gliomacurrent status and future strategies. Childs Nerv Syst 2011 Sep;27(9):1391-7.

[2] Jansen MH, Veldhuijzen van Zanten SE, Sanchez Aliaga E, Heymans MW Warmuth-Metz M, Hargrave D, et al. Survival prediction model of children with diffuse intrinsic pontine glioma based on clinical and radiological criteria. Neuro Oncol 2014 Jun 5.

[3] Slatkin DN, Spanne P, Dilmanian FA, Sandborg M. Microbeam radiation therapy. Med Phys 1992 Nov-Dec;19(6):1395-400.

[4] Schweizer PM, Spanne P, Di Michiel M, Jauch U, Blattmann H, Laissue JA. Tissue lesions caused by microplanar beams of synchrotron-generated X-rays in Drosophila melanogaster. Int J Radiat Biol 2000 Apr;76(4):567-74.

[5] Dilmanian FA, Morris GM, Le Duc G, Huang X, Ren B, Bacarian T, et al. Response of avian embryonic brain to spatially segmented X-ray microbeams. Cell Mol Biol (Noisy-le-grand) 2001 May;47(3):485-93.

[6] Laissue JA, Bartzsch S, Blattmann H, Brauer-Krisch E, Bravin A, Dallery D, et al. Response of the rat spinal cord to X-ray microbeams. Radiother Oncol 2013 Jan;106(1):106-11.

[7] van der Sanden B, Brauer-Krisch E, Siegbahn EA, Ricard C, Vial JC, Laissue J. Tolerance of arteries to microplanar X-ray beams. Int J Radiat Oncol Biol Phys 2010 Aug 1;77(5):1545-52.
[8] Serduc R, Christen T, Laissue J, Farion R, Bouchet A, Sanden B, et al. Brain tumor vessel response to synchrotron microbeam radiation therapy: a shortterm in vivo study. Phys Med Biol 2008 Jul 7;53(13):3609-22.

[9] Serduc R, Verant P, Vial JC, Farion R, Rocas L, Remy C, et al. In vivo two-photon microscopy study of short-term effects of microbeam irradiation on normal mouse brain microvasculature. Int J Radiat Oncol Biol Phys 2006 Apr 1;64(5): $1519-27$.

[10] Zhong N, Morris GM, Bacarian T, Rosen EM, Dilmanian FA. Response of rat skin to high-dose unidirectional X-ray microbeams: a histological study. Radiat Res 2003 Aug; 160(2):133-42.

[11] Slatkin DN, Spanne P, Dilmanian FA, Gebbers JO, Laissue JA. Subacute neuropathological effects of microplanar beams of X-rays from a synchrotron wiggler. Proc Natl Acad Sci U S A 1995 Sep 12;92(19):8783-7.

[12] Bouchet A, Bidart M, Miladi I, Le Clec'h C, Serduc R, Coutton C, et al. Characterization of the 9L gliosarcoma implanted in the Fischer rat: an orthotopic model for a grade IV brain tumor. Tumour Biol 2014 Jul;35(7):6221-33.

[13] Laissue J, Blattmann H, Di Michiel M, Slatkin DN, Lyubimova N, Guzman R, et al. The weanling piglet cerebellum: a surrogate for tolerance to MRT (microbeam radiation therapy) in pediatric neuro-oncology. Proc SPIE 2001;4508:65-73.

[14] Laissue JA, Blattmann H, Wagner HP, Grotzer MA, Slatkin DN. Prospects for microbeam radiation therapy of brain tumours in children to reduce neurological sequelae. Dev Med Child Neurol 2007 Aug;49(8):577-81.

[15] Hanson AL, Slatkin DN, Laissue J. Unidirectional X-ray microbeam radiosurgery of infantile neuraxial malignancies: estimations of tolerable valley doses. Proc SPIE 2013:8565.

[16] Laissue J, Lyubimova N, Wagner HP, Archer DW, Slatkin DN, Di Michiel M, et al. Microbeam radiation therapy. Proc SPIE 1999;3770:38-45.

[17] Bouchet A, Lemasson B, Christen T, Potez M, Rome C, Coquery N, et al. Synchrotron microbeam radiation therapy induces hypoxia in intracerebral gliosarcoma but not in the normal brain. Radiother Oncol 2013 Jul;108(1): 143-8.

[18] Dilmanian FA, Qu Y, Feinendegen LE, Pena LA, Bacarian T, Henn FA, et al Tissuesparing effect of X-ray microplanar beams particularly in the CNS: is a bystander effect involved? Exp Hematol 2007 Apr;35(4 Suppl. 1):69-77.

[19] Fernandez-Palomo C, Schultke E, Smith R, Brauer-Krisch E, Laissue J Schroll C, et al. Bystander effects in tumor-free and tumor-bearing rat brains following irradiation by synchrotron X-rays. Int J Radiat Biol 2013 Jun;89(6): 445-53.

[20] Smith RW, Wang J, Schultke E, Seymour CB, Brauer-Krisch E, Laissue JA, et al. Proteomic changes in the rat brain induced by homogenous irradiation and by the bystander effect resulting from high energy synchrotron X-ray microbeams. Int J Radiat Biol 2013 Feb;89(2):118-27.

[21] Mothersill C, Fernandez-Palomo C, Fazzari J, Smith R, Schultke E, BrauerKrisch E, et al. Transmission of signals from rats receiving high doses of microbeam radiation to cage mates: an inter-mammal bystander effect. Dose Response 2014 Jan;12(1):72-92.

[22] Bouchet A, Boumendjel A, Khalil E, Serduc R, Brauer E, Siegbahn EA, et al Chalcone JAI-51 improves efficacy of synchrotron microbeam radiation therapy of brain tumors. J Synchrotron Radiat 2012 Jul;19(Pt 4):478-82.

[23] Schultke E, Juurlink BH, Ataelmannan K, Laissue J, Blattmann H, BrauerKrisch E, et al. Memory and survival after microbeam radiation therapy. Eur J Radiol 2008 Dec;68(3 Suppl.):S142-6.

[24] Griffin RJ, Koonce NA, Dings RP, Siegel E, Moros EG, Brauer-Krisch E, et al. Microbeam radiation therapy alters vascular architecture and tumor oxygenation and is enhanced by a galectin- 1 targeted anti-angiogenic peptide. Radiat Res 2012 Jun;177(6):804-12.

[25] Le Duc G, Miladi I, Alric C, Mowat P, Brauer-Krisch E, Bouchet A, et al. Toward an image-guided microbeam radiation therapy using gadolinium-based nanoparticles. ACS Nano 2011 Dec 27;5(12):9566-74.

[26] Smilowitz HM, Blattmann H, Brauer-Krisch E, Bravin A, Di Michiel M Gebbers JO, et al. Synergy of gene-mediated immunoprophylaxis and microbeam radiation therapy for advanced intracerebral rat 9L gliosarcomas. J Neurooncol 2006 Jun;78(2):135-43.

[27] Serduc R, Brauer-Krisch E, Siegbahn EA, Bouchet A, Pouyatos B, Carron R, et al. Highprecision radiosurgical dose delivery by interlaced microbeam arrays of high-flux low-energy synchrotron X-rays. PLoS One 2010;5(2):e9028.

[28] Bouchet A, Lemasson B, Le Duc G, Maisin C, Brauer-Krisch E, Siegbahn EA, et al. Preferential effect of synchrotron microbeam radiation therapy on intracerebral 9L gliosarcomas vascular networks. Int J Radiat Oncol Biol Phys 2010 Dec 1;78(5):1503-12.

[29] Bouchet A, Sakakini N, El Atifi M, Le Clec'h C, Brauer E, Moisan A, et al. Early gene expression analysis in 9L orthotopic tumor-bearing rats identifies immune modulation in molecular response to synchrotron microbeam radiation therapy. PLoS One 2013;8(12):e81874.

[30] Sprung CN, Yang Y, Forrester HB, Li J, Zaitseva M, Cann L, et al. Genome-wide transcription responses to synchrotron microbeam radiotherapy. Radiat Res 2012 Oct; $178(4): 249-59$.

[31] Selek L, Seigneuret E, Nugue G, Wion D, Nissou MF, Salon C, et al. Imaging and histological characterization of a human brain xenograft in pig: the first induced glioma model in a large animal. J Neurosci Methods 2014 Jan 15;221: 159-65.

[32] Lindblad-Toh K, Wade CM, Mikkelsen TS, Karlsson EK, Jaffe DB, Kamal M, et al. Genome sequence, comparative analysis and haplotype structure of the domestic dog. Nature 2005 Dec 8;438(7069):803-19. 
[33] Khanna C, Lindblad-Toh K, Vail D, London C, Bergman P, Barber L, et al. The dog as a cancer model. Nat Biotechnol 2006 Sep;24(9):1065-6.

[34] Vail DM. Cancer clinical trials: development and implementation. Vet Clin North Am Small Anim Pract 2007 Nov;37(6):1033-57.

[35] MacEwen EG. Spontaneous tumors in dogs and cats: models for the study of cancer biology and treatment. Cancer Metastasis Rev 1990 Sep;9(2):125-36.
[36] Stupp R, Mason WP, van den Bent MJ, Weller M, Fisher B, Taphoorn MJ, et al. Radiotherapy plus concomitant and adjuvant temozolomide for glioblastoma. N Engl J Med 2005 Mar 10;352(10):987-96.

[37] Uh J, Merchant TE, Li Y, Li X, Hua C. MRI-based treatment planning with pseudo CT generated through atlas registration. Med Phys 2014 May;41(5): 051711. 